\begin{tabular}{|c|c|}
\hline Title & A re numerical weather model outputs hel pful to reduce tropospheric delay signals in InSA R data? \\
\hline Author(s) & Kinoshita, Y ouhei; Furuya, Masato; Hobiger, Thomas; Ichikawa, Ryuichi \\
\hline Citation & $\begin{array}{l}\text { Journal of Geodesy, 87(3), 267-277 } \\
\text { https://doi.org/10.1007/s00190-012-0596-x }\end{array}$ \\
\hline Issue Date & $2013-03$ \\
\hline Doc URL & http:/hdl .handle.net/2115/54772 \\
\hline Rights & The original publication is available at www.springerlink.com \\
\hline Type & article (author version) \\
\hline Additional Information & There are other files related to this item in HUSCAP. Check the above URL. \\
\hline File Information & JoG87-3_267-277.pdf \\
\hline
\end{tabular}

Instructions for use 


\title{
Are numerical weather model outputs helpful to reduce tropospheric delay signals in InSAR data?
}

\author{
Youhei Kinoshita - Masato Furuya - Thomas Hobiger • Ryuichi Ichikawa
}

Received: date / Accepted: date

\begin{abstract}
Interferometric Synthetic Aperture Radar (InSAR) phase data include not only signals due to crustal movements but also those associated with microwave propagation delay through the atmosphere. In particular, the effect of water vapor can generate apparent signals on the order of a few centimeters or more, and prevent us from detecting such geophysical signals as those due to secular crustal deformation. In order to examine if and to what extent numerical weather model (NWM) outputs are helpful to reduce the tropospheric delay signals at spatial scales of $5 \sim 50 \mathrm{~km}$ wavelengths, we compared three approaches of tropospheric signal reduction, using 54 interferograms in central Hokkaido, Japan. The first approach is the conventional topography-correlated delay correction that is based on the regional digital elevation model (DEM). The second approach is based on the Japan Meteorological Agency's operational meso-scale analysis model (MSM) data, where we compute tropospheric delays and subtract them from the interferogram. However, the MSM data are available at predefined epochs, and their spatial resolution is about $10 \mathrm{~km}$, and therefore we need to interpolate both temporally and spatially to match with interferograms. Expecting to obtain a more physically plausible reduction of the tropospheric effects, we ran a 1-km mesh high-resolution numerical weather model WRF (Weather Research and Forecasting model) by ourselves, using the MSM data as the
\end{abstract}

Y. Kinoshita and M. Furuya

Graduate School of Sciences, Hokkaido University, N10W8, Kita-ku, Sapporo, Hokkaido 060-0810, Japan

Tel. \& Fax: +81-11-706-2759

E-mail: youhei_skt@mail.sci.hokudai.ac.jp

T. Hobiger and R. Ichikawa

National Institute of Information and Communications Technology, Nukui-kita, Koganei, Tokyo 184-8795, Japan initial and boundary conditions. The third approach is similar to the second approach except that we make use of the WRF-based tropospheric data.

Results show that if the topography-correlated phases are significant, both the conventional DEMbased approach and the MSM-based approach reveal comparable performances. However, when the topography-correlated phases are insignificant, none of the approaches could efficiently reduce the tropospheric phases. Although it could reduce the tropospheric signals in a local area, in none of the case studies did the WRF model produce the "best" performance. Whereas the global atmospheric model outputs are shown to be effective in reducing long-wavelength tropospheric signals, we consider that further improvements are needed for the initial and boundary condition data for highresolution NWM, so that the NWM-based approach will become more reliable even in the case of a nonstratified troposphere.

Keywords InSAR · tropospheric delay · numerical weather model · ALOS

\section{Introduction}

Interferometric Synthetic Aperture Radar (InSAR) allows us to measure surface displacements from the phase differences at two acquisition dates after correcting for orbital contribution and surface topography. Ever since the detection reports of the co-seismic deformation due to the 1992 Landers earthquake (Massonnet et al. 1993) and the glacier movements at Antarctica (Goldstein et al. 1993), InSAR has been applied to a variety of phenomena such as volcano deformation, ground subsidence due to water pumping, strain accumulation around plate boundaries, and post-seismic de- 
formation (e.g., Massonnet and Feigl, 1998; Bürgmann et al., 2000; Hanssen, 2001; Simons and Rosen, 2009; Furuya, 2011). However, InSAR cannot detect every small-amplitude signal due to the presence of errors. In particular, microwave propagation delays caused by the troposphere can mask small-amplitude deformation signals with spatial scales longer than $\sim 10 \mathrm{~km}$.

Microwave propagation delays consist of those originated in the ionosphere and the troposphere, the latter of which further consists of those due to water vapor, dry air and liquid water content (e.g. Thayer, 1974; Wadge et al. 2010). Hydrostatic delay and wet delay can be computed from pressure, temperature, and water vapor distribution data using empirical relations (e.g., Hanssen, 2001). As for the troposphere, the magnitude of the hydrostatic delay is greater than that of the wet delay which amounts to $\sim 20 \%$ at maximum. For the InSAR-based deformation measurements, however, the difference between the total delays at two acquisition epochs is observed, and the effect of wet delay becomes larger, because the hydrostatic delay is largely dependent upon surface air pressure that is nearly constant over time, and will tend to cancel out at two different epochs (Zebker et al., 1997); in mountainous areas, however, hydrostatic delays could become significant (Elliott et al., 2008). While the wet delay is smaller in terms of its amplitude, water vapor distribution is highly variable in both space and time, and its characteristic spatial scale overlaps with that of crustal deformation. The wet delay, therefore, can appear as an artifact of a crustal deformation signal.

To isolate small-amplitude crustal deformation signals from the interferogram, several approaches have been developed to correct for, or at the least reduce the tropospheric delays. In the stacking approach, advantage is taken from the spatio-temporal characteristics of a random troposphere and underlying persistent crustal deformation. Thus, one can sum up a large number of interferograms to reduce tropospheric effects and to detect crustal deformation signals (e.g., Fujiwara et al., 1998; Furuya et al., 2007). The stacking approach, however, is valid mainly for the detection of secularly steady deformation signals. Meanwhile, we often encounter that phase is highly correlated with the regional topography even after the subtraction of topographic phases (e.g. Elliot et al., 2008; Fournier et al., 2011). Those phases can be interpreted as the differences in the water vapor distribution over rugged terrain between the two epochs, and are also called "stratified" delay. Taking advantage of the high-resolution digital elevation model (DEM), we perform a simple linear or quadratic regression analysis to model and subtract the topography-correlated phases (e.g., Fujiwara et al.,
1999). Lin et al. (2010) proposed a scale-dependence in the topography-correlated signals, decomposed the DEM into several spectral components, and performed the similar regression analysis at each spectral component. In the time series analysis techniques such as the Permanent (Persistent) Scatterer (Ferreti et al., 2000; Hooper et al., 2004) and the Small Baseline Subset (Berardino et al., 2002), the tropospheric phases which are random in time are reduced, by applying a low-pass filter along the temporal axis since they will behave temporally random. Doin et al. (2009) emphasized the tropospheric delay corrections prior to a stacking or a timeseries anaysis in order to eliminate biases in long-term strain rate estimates. The calibration-type approaches employ the other independent data to correct for the tropospheric signals. Onn and Zebker (2006) proposed an approach to exploit the zenith wet delay data derived from a regional GPS network. Li et al. (2005, 2006) employed MERIS, MODIS and GPS water vapor data to estimate and reduce the tropospheric signals in the interferogram. The problem with using GPS is their sparse spatial distribution. Also, MERIS and MODIS data are not always available at the time of the SAR data acquisitions.

Another approach is to compute tropospheric delays, using the output data from a numerical weather model (NWM), and subtract them from the interferogram (e.g. Shimada, 1999; Otsuka et al., 2002; Wadge et

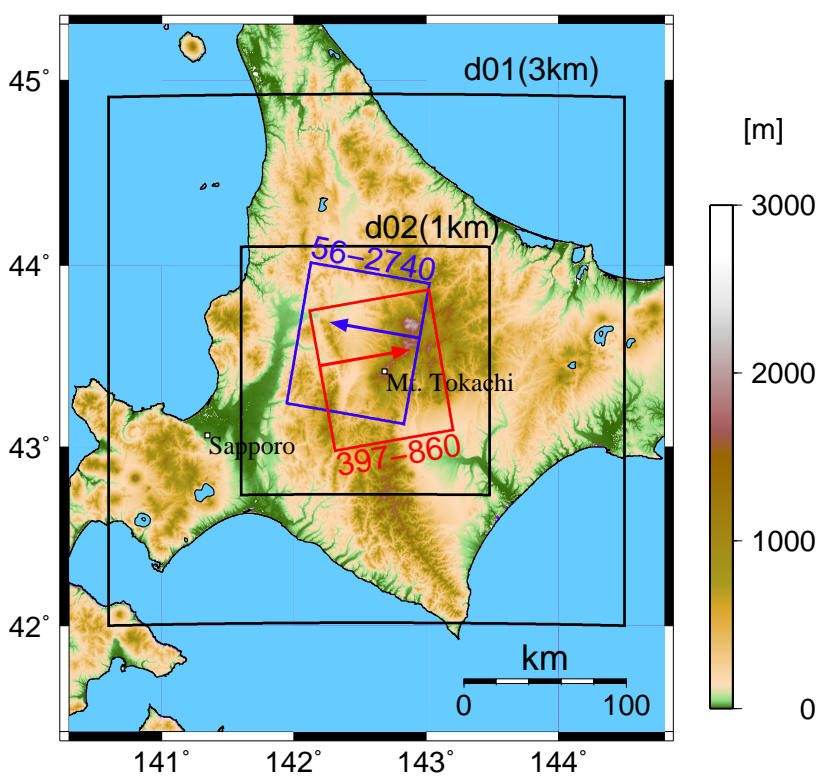

Fig. 1 Location of the study area. Red and blue rectangle represents the ascending and descending tracks, respectively. Red and blue vectors represent the radar line-of-sight direction. Two black rectangles represent domain 1 (3 km gridspacing) and domain 2 (1 km grid-spacing) of WRF respectively. 
al., 2002; Foster et al., 2006; Ozawa and Shimizu, 2010; Jolivet et al. 2011). Shimada (1999) and Ozawa and Shimizu (2010) used a 1.25-degree-mesh Global Analysis (GANAL) model and 10-km-mesh Meso Scale Model (MSM) of Japan Meteorological Agency (JMA), respectively, and concluded that they were "effective" in reducing tropospheric effects. A similar approach was undertaken by Jolivet et al. (2011), who employed the 75km-mesh ERA-Interim model from European Center for Medium-Range Weather Forecast. However, even in the recent JMA/MSM data, the spatial resolution of operational analysis is $10 \mathrm{~km}$, which is orders-ofmagnitude coarser than actual interferograms and evidently unable to reproduce the finer-scale tropospheric signals that are not correlated with the topography.

Moreover, those operational meteorological data were computed at several fixed epochs in a day, which are usually different from the acquisition times of SAR data by more than an hour. Those operational analysis data are used directly or temporally interpolated to derive the data at the time of the data acquisition. Other studies therefore performed their own NWM computation, using even higher resolution model (e.g., Otsuka et al., 2002; Wadge et al., 2002, 2010; Foster et al., 2006; Puysségur et al., 2007). Wadge et al (2010) tried to perform the tropospheric delay correction, using a 0.3 km-resolution NWM, but concluded that model results were still inaccurate due to the coarse representation of initial conditions.

The purpose of this paper is to compare the performance of three approaches of tropospheric delay correction, two of which are based on the NWM data, using 54 interferograms formed from 26 acquisition dates in Hokkaido, Japan (Figure 1). The first approach is a simple DEM-based linear regression, and the second approach is based on the operational NWM output. While Shimada (1999) and Ozawa and Shimizu (2010) tested this approach around Mt. Fuji area, Japan, we examine the MSM-based approach at other mountainous areas, where the elevation differences are highly variable from $100 \mathrm{~m}$ to $2300 \mathrm{~m}$. In the third approach, we use a state-of-the-art high-resolution non-hydrostatic numerical weather forecasting model, Weather Research and Forecasting (WRF) model (Skamarock et al., 2008), and compute the tropospheric delays at the radar acquisition time with spatial resolution of $1 \mathrm{~km}$. In the second and third approach, we take advantage of the Kashima Ray-Tracing Tool (KARAT, Hobiger et al., 2008) so that we can more realistically perform the computation of tropospheric delays; see Hobiger et al. (2010) for the comparison of ray-tracing approach with the conventional mapping approach. In this study, we do not intend to show the best performance of any ap- proach but to compare and discuss the performance and limitation of the present state-of-the-art approaches.

While global analysis model outputs are shown to be useful to reduce long-wavelength tropospheric signals (e.g., Doin et al., 2009; Jolivet et al., 2011), we focus our attention to the delays with shorter wavelengths. This is partly because both orbit errors and ionosphere can generate long-wavelength noises, which we remove by polynomial fitting in the following analyses, and partly because we can examine the impact of higher-resolution NWM outputs.

\section{InSAR and Tropospheric delay reduction}

\subsection{Microwave propagation delay}

The microwave propagation delay $L_{e}$ from the surface to the satellite is expressed as the difference of the actual propagation length in the atmosphere from that in the vacuum, namely,

$L_{e}=\int_{a t m}(n(\mathbf{r})-1) d s+\int_{a t m} d s-\int_{v a c} d s$,

where $n$ is refractive index at position $\mathbf{r}$, and the integration is performed along the propagation path $s$. The first term represents the electromagnetic delay and the second and third term represents the effect of raybending. The refractivity of the neutral atmosphere $N$ can be expressed as (e.g. Thayer, 1974; Hanssen, 2001; Puysségur et al., 2007),

$N=10^{-6}(n-1)=k_{1} \frac{P_{d}}{T}+k_{2} \frac{P_{v}}{T}+k_{3} \frac{P_{v}}{T^{2}}$,

where $P_{d}$ is the dry partial air pressure in Pascal $(P a)$, $P_{v}$ is the water vapor partial pressure in $P a$ and $T$ is the temperature in Kelvin $(K)$. We derived these three parameters from MSM and WRF. In this paper we set the three constants according to Bevis et al. (1994), i.e., $k_{1}=77.604\left(K h P a^{-1}\right), k_{2}=70.4\left(K h P a^{-1}\right)$, and $k_{3}=373.900\left(K^{2} h P a^{-1}\right)$, respectively. Thus the three dimensional refractivity field at the time of SAR acquisition is obtained from equation (2). Finally, instead of using a mapping function approach, total atmospheric path delay at each pixel is calculated with KARAT based on ray tracing method (Hobiger et al., 2010).

In many previous studies, tropospheric delay has been considered as the sum of two components, hydrostatic delay and wet delay, and they have been estimated separately. The hydrostatic delay was modeled as a function of latitude, height and surface pressure (e.g. Elgered, 1993). However, it is derived on the assumption of a hydrostatic approximation in the pressure profile, and hence the zenith total delay is first 
computed. The zenith total delay is then converted to the slant range delay with a use of a mapping function. Although this approach provides us with a good approximation of the actual delay, we adopt a more rigorous ray-tracing approach without using a mapping function (see Hobiger et al. 2010 for details).

\subsection{InSAR processing}

Interferograms were generated from the ALOS/PALSAR level 1.0 data with the use of Gamma SAR processor. Table S1 is a list of the SAR data pairs used in this paper. Figure 1 shows our study area in the middle of Hokkaido, Japan. With the exception of the very localized signal at the Mt. Tokachi volcano (Hobiger et al., 2010), no significant large-scale crustal deformations were expected during the analyzed period. The deformation rate and area are not large enough to generate measurable effects on our analyzed data. To correct for the topographic fringes, we use the 10-meter mesh digital elevation model (DEM) by the Geospatial Information Authority of Japan (GSI). The spatial resolution of each multi-looked interferogram is $\sim 80 \mathrm{~m}$ in range and azimuth. Phase unwrapping was performed on multi-looked interferograms after applying adaptive spectral filtering with a strength exponent 0.6 and a filtering window size of $32 \times 32$ pixels (Goldstein \& Werner, 1998).

The orbital contribution is removed with the use of precision orbit data by JAXA, which we do not reestimate further. Nonetheless, long-wavelength residual phases sometimes remain in the initial interferogram, which could be due to either orbit errors, tropospheric delays, ionospheric delays, or a combination of these effects. However, as we cannot accurately quantify each contribution of these long-wavelength noises, we simply subtract any long-wavelength phases by fitting with second-order polynomials in both range and azimuth direction. Although this procedure surely prevents us from detecting long-wavelength smallamplitude tectonic deformation signals, we do not aim in this study to detect such signals. In the following MSM- and WRF-based approach, however, the removal of long-wavelength polynomials was performed after the subtraction of NWM-based corrections, because the NWM-based models include their own longwavelength phases. Although the long-wavelength tropospheric model could be useful for long-wavelength deformation studies (Fournier et al., 2011; Jolivet et al., 2011), we removed them to perform fair comparison at small- to medium-wavelength scales.

\subsection{Topography-correlated delay correction}

Topography-correlated delay correction is readily performed by a simple linear regression analysis on the unwrapped differential phases with the aid of a DEM, and is capable of efficiently reducing the tropospheric artifacts (e.g., Fujiwara et al., 1999; Cavalié et al, 2008; Ozawa and Shimizu, 2010). To compare the results with those derived from NWM-based approaches, we also assume that the tropospheric phase is a linear function of the local elevation and an additional constant. We derive the two coefficients, $a_{\text {topo }}$ that is proportional to the elevation and a constant $a_{\text {const }}$, and apply these to the InSAR phases every 32 pixels along range and azimuth directions. The corrected interferogram $\phi_{t c}$ can be represented as follows:

$\phi_{t c}=\phi_{\text {org }}-\left(a_{\text {const }}+a_{\text {topo }} H\right)$,

where $\phi_{\text {org }}$ and $H$ are the interferogram phase after removal of long-wavelength polynomial phases and the local elevation, respectively.

\subsection{MSM-based approach}

In order to examine how effective the operational NWM output is, we compute tropospheric delays, using the temperature, pressure, and water vapor data from the JMA/MSM gridded data.

The JMA/MSM data are available every three hours a day. Ozawa and Shimizu (2010) used the data at two nearby epochs to linearly interpolate the data at the instant of SAR data acquisition, and we followed the same procedure. The spatial resolution of JMA/MSM is 10 $\mathrm{km}$, and at 16 constant pressure levels along the vertical axis. Because the ray-tracing of tropospheric delay has to be performed along the radar line of sight, we have to know the actual ellipsoidal height of the surface at a spatial resolution comparable to the interferogram. Because of the coarse resolution of the MSM, we obviously need to perform spatial interpolation not only along the horizontal but also along the vertical directions. Consequently, as in all previously published NWM-based approaches, the MSM-based correction is dependent on the fine-resolution digital ellipsoidal-height model as well.

In order to match the spatial resolution of the interferogram, KARAT first re-grids and interpolates the NWM data (Hobiger et al., 2008). The KARAT searches for 8 grid points in the original MSM data that enclose any arbitrary starting point in the interferogram. Thereby, the lower 4 points are from the closest layer below the point, and the upper 4 points are 

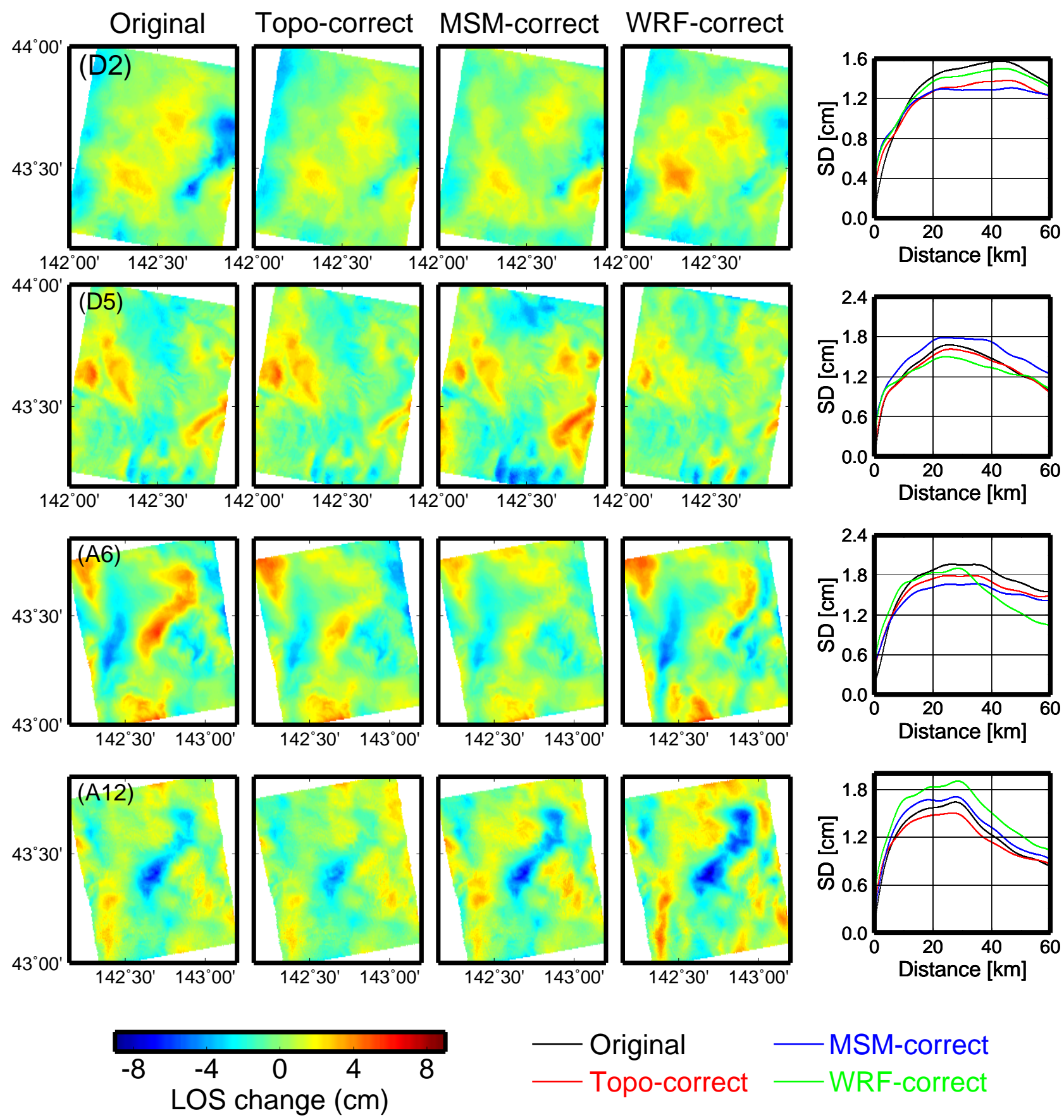

Fig. 2 Four original and corrected interferograms and their variograms. (Left column) Original interferogram after removal of long-wavelength phases, (Second column from left) Topography-correlated delay correction based on DEM, (Third column from left) MSM-based correction, (Fourth column from left) WRF-based correction, (Fifth column from left) the variograms of four interferograms. Details of each pair are listed in Table S1.

from the nearest layer above the point. Once these $2 \times 4$ points are found, a bilinear interpolation is performed to obtain a value at the desired point at each layer. Finally, a vertical exponential interpolation is performed to get the refractivity at the point, thereby the raytracing starts to derive the tropospheric delay. A ver- tical interpolation based on the grid nodes, however, might introduce significant artifacts where the weather model's DEM differs significantly from the real topography. Nevertheless, in the case of our study, at only less than 0.5 percent of the total pixels the weather model's DEM differ by more than $200 \mathrm{~m}$ from the DEM used 
to generate interferograms. Therefore the issue about a vertical interpolation doesn't affect our results and conclusions. Setting the delay estimates for the master and slave image as $D_{m}$ and $D_{s}$, the MSM-based corrected interferogram $\phi_{m s m}$ becomes as follows;

$\phi_{m s m}=\phi_{i n t}-\left(D_{m}-D_{s}\right)$.

where $\phi_{\text {int }}$ denotes the initial interferogram.

As stated before, the $\left(D_{m}-D_{s}\right)$ in eq. (4) also includes long-wavelength phases. Thus, after subtracting $\left(D_{m}-D_{s}\right)$ from $\phi_{i n t}$, we remove the long-wavelength phases by the polynomial fitting to the $\phi_{m s m}$. The same procedure is performed in the following WRF-based correction, too.

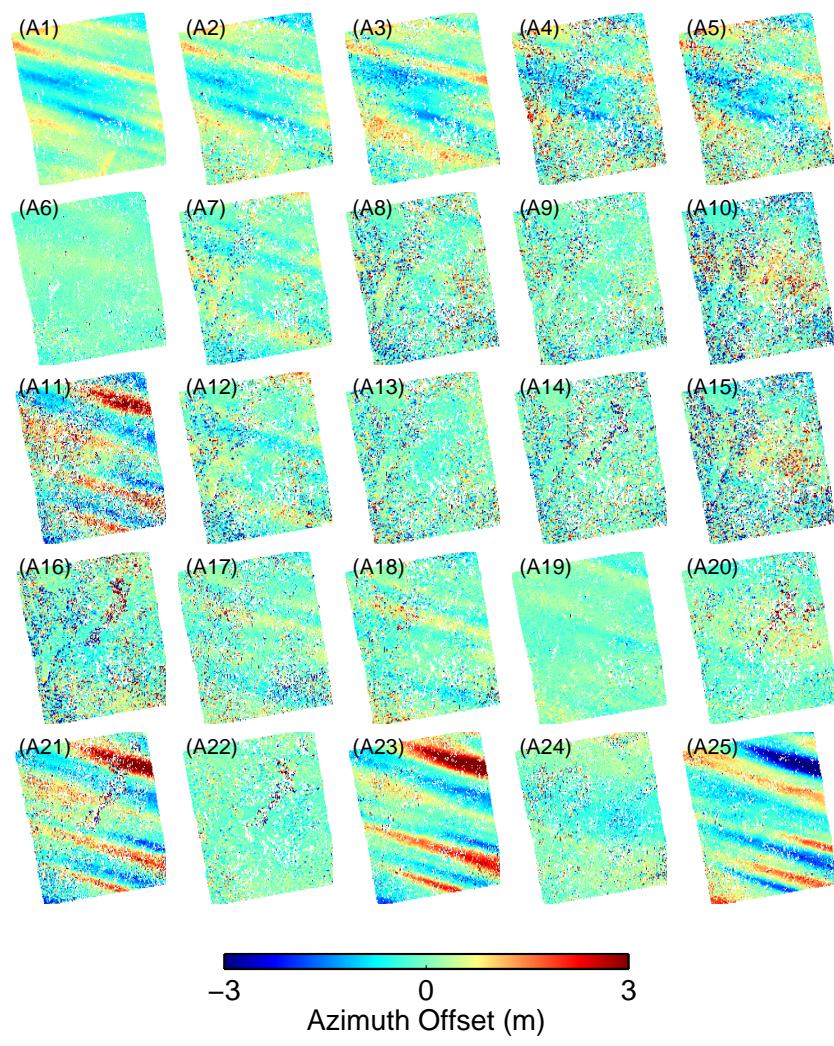

Fig. 3 Azimuth offsets of ascending orbit data. Details of each pair are listed in Table S1.

\subsection{WRF-based approach}

The MSM data are much coarser than the interferogram in terms of their spatial resolution, and are available only 8 times a day. The WRF model can produce spatially denser tropospheric data at the instant of the SAR data acquisition with the expectation that the WRF would provide a more realistic troposphere.
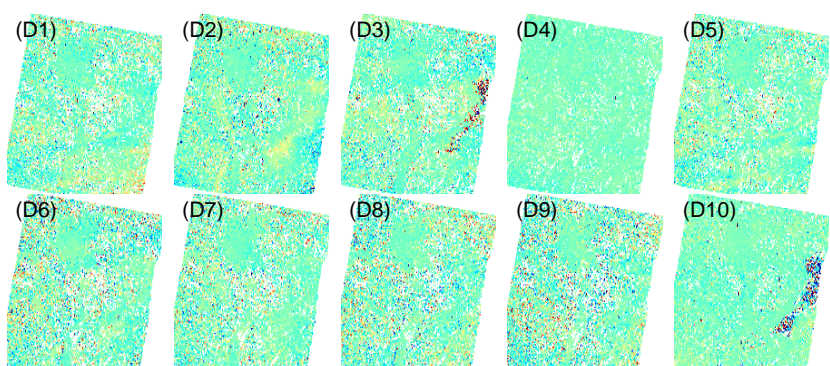

(D10)
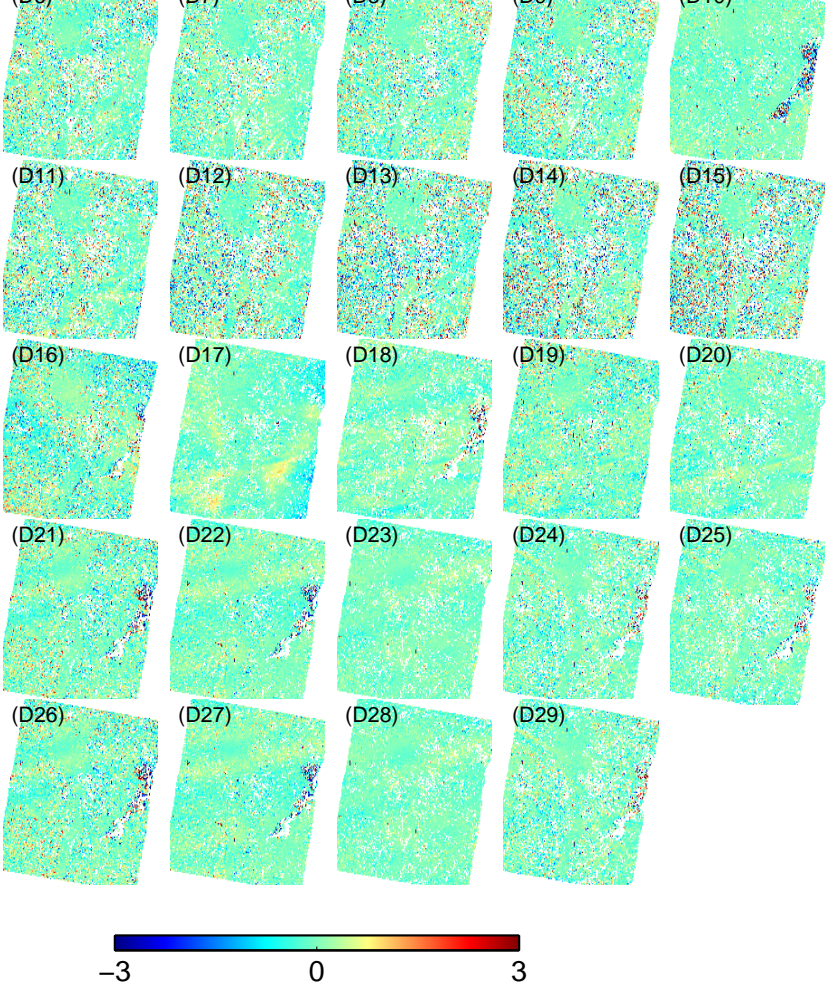

\section{Azimuth Offset $(\mathrm{m})$}

Fig. 4 Azimuth offsets of descending orbit data. Details of each pair are listed in Table S1.

WRF is a non-hydrostatic numerical weather prediction model mainly developed at U. S. National Center for Atmospheric Research (NCAR) and National Center for Environmental Prediction (NCEP), and is the next generation model of the MM5 which has been used by Foster et al. (2006) and Puysségur et al. (2007) for similar studies. Employing the output data of JMA/MSM as the initial and boundary conditions for the WRF, we perform the numerical integration. Here, the numerical integration consists of two nested domains increasing in resolution $(3,1 \mathrm{~km}$ and shown in Fig 1. For the topography in the NWM, we use GTOPO30 with a horizontal resolution of $1 \mathrm{~km}$, which is still coarser than that of the interferogram. In the vertical direction, there are 60 layers from the ground surface to the $10 \mathrm{hPa}$ level ( $\sim 30 \mathrm{~km}$ altitude). Since some parameter data such as soil moisture are not provided in the JMA/MSM data, we complement them with those available from the global objective analysis data by U.S. NCEP (http://dss.ucar.edu/datasets/ds083.2/). Although it is possible to change a variety of param- 
eter settings in the WRF, we set all the parameters as default values. The prediction performance of the WRF is shown be comparable to that of JMA's NonHydrostatic Model at the spatial resolution of $20 \mathrm{~km}$ (Hayashi et al., 2008; Chan et al., 2010), but an intercomparison of non-hydrostatic models is beyond the scope of this paper. As done in the MSM-based reduction, the original WRF data are re-gridded and interpolated to match the spatial resolution of the interferogram. The corrected interferogram $\phi_{W R F}$ becomes as follows;

$\phi_{W R F}=\phi_{i n t}-\left(D_{m}^{\prime}-D_{s}^{\prime}\right)$.

\section{Results and Discussion}

The 54 original and corrected interferograms are shown in Figure S1. In the main text, we show selected four original and corrected interferograms in Figure 2. We observe that all the data pairs include signals correlated with the local topography although the extent of correlation varies in each case. The peak-to-peak amplitude of the topography-correlated signals can reach 10 20 $\mathrm{cm}$ changes along the radar line-of-sight (LOS). In addition to the topography-correlated signals, we also notice broad signals with the spatial scale of $5 \sim 50 \mathrm{~km}$ that are not correlated with the topography. We consider that most of them are due to the heterogeneous tropospheric delays.

However, in the case of L-band SAR data, the effect of ionospheric signal becomes significant as compared to the C-band and X-band SAR data (e.g. ERS, ENVISAT, and TerraSAR-X), and may be a non-negligible error source in some of the ALOS/PALSAR interferograms (Shimada et al., 2008). We can judge the ionospheric effects on the interferogram from the azimuth offset data since spatial variations in the free electron density in the ionosphere can cause azimuth positional shifts and are observed as "azimuth streak" in the azimuth offset data (Gray et al., 2000, Meyer et al., 2006). Figure 3 and 4 show the azimuth offset data of ascending and descending orbit, respectively (see Table S1). The clearer streaks in the ascending data probably appear because the PALSAR data are acquired at the local time around 22:30, when the ionospheric disturbances are larger than during the day (descending data). However, because the impact of ionospheric disturbances on the interferogram is evaluated from an integration of the azimuth offset along the azimuth (flight) direction (Meyer et al., 2006), the ionospheric effects will generate longer-wavelength phases in the interferogram that were mostly taken out by the removal with the polynomial fitting. In this study, 9 azimuth offset data (Figure 3, A1-A5, A11, A21, A23 and A25) show clear streaks due to the ionosphere, and interferograms of those data reveal larger long-wavelength phase disturbances than other data. Consequently, the effects of ionosphere on some but not all of the original ascending InSAR data are not negligible.

The second column in Figure 2 and S1 shows interferograms after the topography-correlated delay correction. We notice that the topography-correlated signals were notably reduced particularly for 19 interferograms (D2, D5, A6 and A12 in Figure 2, and D4, D7-D9, D17, D18, D21, D23, A2, A5, A9, A14, A15, A18, and A19 in Figure S1), each of which included large apparent range changes around the mountain areas (see first column in Figures 2 and S1). However, we also observe that there still remain signals that are correlated with the topography with amplitudes reaching $\pm 5 \mathrm{~cm}$, for instance in D8, D9, and D23. In Figure 2 A6, for example, the areas of positive range changes around the mountain areas clearly decreased with topography-correlated delay correction, but those negative changes to the southeast of the mountain areas do not change appreciably. The first-order polynomial regression as expressed by eq. (3) might be not optimal for the entire scene, and higher-order polynomial regression may be necessary. More elaborate DEM-based approaches may be able to reduce the apparent signals (e.g., Lin et al. 2010). However, as we often aim to detect crustal deformation signals that are correlated with the regional topography, which is often the case, for instance, at volcanoes (Beauducel et al. 2000) and along major faults (Elliot et al. 2008), and thus the DEM-based approaches should be applied very carefully in order not to take out the desired deformation signals.

The third column of Figure 2 and S1 shows interferograms after the MSM-based delay correction. In 11 interferograms (D2 and A6 in Figure 2, and D17, D19, D21, D24, A2, A5, A9, A19, and A24 in Figure S1), we observe that the large amplitude signals in original interferograms could be efficiently reduced, particularly over the mountain areas. Especially for D19 and A6, the MSM-based approach reduces the signals more efficiently than the DEM-based approach. However, we also acknowledge that in some cases there arises other apparent LOS changes on the order of $\sim 5 \mathrm{~cm}$ that were absent in original interferograms. For instance, those distributed in the mountain areas in D3 of Figure S1, to the north and to the south in D26 and to the middle of A17 were all generated after the MSM-based correction. It is thus evident that the MSM-based approach does not always eliminate the tropospheric signals. Additionally, in A24 the positive signals in the middle to the north-east of the original interferogram were absent 
after the MSM-based correction, but arose around the north-west corner and to the south-east with a scale of $\sim 20 \mathrm{~km}$ that were absent in the original interferogram. Since those signals are not apparently correlated with the topography, we consider that they could represent some non-stratified heterogeneous signals and would be artifacts due to the errors in the MSM data. The ionospheric influence in A24 seems to be small in light of the small amplitude in the azimuth offset data of A24 in Figure 3.

The fourth column of Figure 2 and S1 shows interferograms after the WRF-based delay correction. Overall, derived interferograms reveal similar signals as those after the MSM-based correction. The topographycorrelated signals in the original interferograms became smaller by WRF-based approach as observed in interferograms after MSM-based correction. However, we need to acknowledge that, even if the WRF-data are derived at the instant of SAR data acquisitions with a spatial scale of $1-\mathrm{km}$, the heterogeneous signals with the spatial scale ranging from 5 to $50 \mathrm{~km}$ may not be efficiently reduced (for example, see D12, D23, A7, and A14 in Figure S1). Although we performed the numerical integration using the WRF model with 1-km spatial scale, we should recall that the initial and boundary conditions for the numerical integration are all based on the MSM and NCEP analysis data whose spatial and temporal resolution is coarser than those in the WRF. As discussed by Wadge et al (2010), we need more accurate data sets for the initial and boundary conditions.

It is evident from Figure 2 and S1 that the amplitude of tropospheric delay signals depends on the spatial scale and tends to become larger as the spatial scale becomes larger. Although it can be simply computed and sometimes useful, a single standard deviation computed from the entire pixel population in the residual interferogram does not represent the multi-scale characteristics in the tropospheric delay signals. In order to quantify the magnitude of the residual tropospheric delays in each interferogram, we use the variogram,

$\gamma(\mathbf{h})=\frac{1}{2}(\phi(\mathbf{x})-\phi(\mathbf{x}+\mathbf{h}))^{2}$

which computes the variance of all pixel pairs at a given distance $h$ and allow us to examine the scale-dependence of the two-dimensional signals (e.g. Hanssen, 2001; Hobiger et al. 2010; Ozawa and Shimizu, 2010).

The fifth column of Figure 2 and S1 shows the square-root of the variogram (standard deviation, SD) for each interferogram with and without the tropospheric delay corrections. In the variograms, a reduction of the SD over the wavelengths of $20 \sim 40 \mathrm{~km}$ is recognized in 20 interferograms for the DEM-based correc-

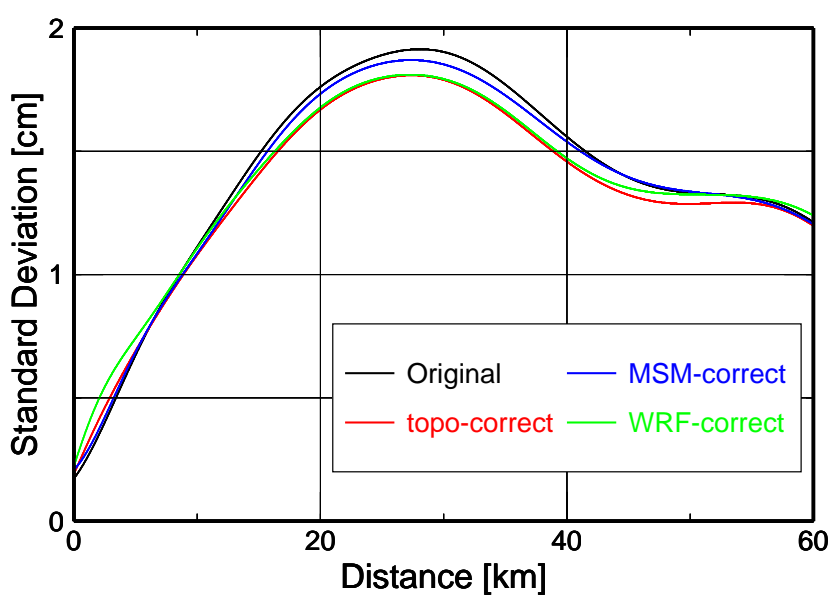

Fig. 5 Variograms of four original and corrected interferograms for all (54) data pairs.

tion, in 9 interferograms for the MSM-based correction and in 15 interferograms for the WRF-based correction. However, we also recognize an SD increase in 25 interferograms for the MSM-based correction and 23 interferograms for the WRF-based correction. It turns out that the performance of each correction approach strongly depends on each interferogram, and that no straightforward conclusions can be drawn regarding the different correction approaches.

Figure 5 shows summarized variograms of four original and corrected interferograms for all 54 data pairs shown in Figure S1. This also indicates that the DEMbased approach is the most effective for correcting original interferograms chosen here. However, the WRFbased approach shows the performance as equivalent as the DEM-based approach over the wavelengths of $20 \sim 40 \mathrm{~km}$, but worse performance over the wavelengths of shoter than $20 \mathrm{~km}$ and longer than $40 \mathrm{~km}$. The amount of SD reduction of the MSM-based approach is smaller than that of other approaches in this study, although an SD reduces in a way.

We examined how the phase dependence with elevation varied after each correction approach. Figures 6 and 7 are examples for D2 and A12, respectively. Whereas the MSM- and WRF-based correction reduce the phase dependences with elevation and the extent of the "non-stratified" phase variation in the interferogram of D2 (Figure 6), those in A12 increase the effect (Figure 7). Figures 8 and 9 summarize the analysis for all interferograms, and indicate how each correction reduced or increased the phase dependences with elevation, by normalizing with the slope of original interferograms. The MSM-based correction often generate larger slopes that in the original signals (29 interferograms in Figs. 8 and 9), indicating that the MSM- 

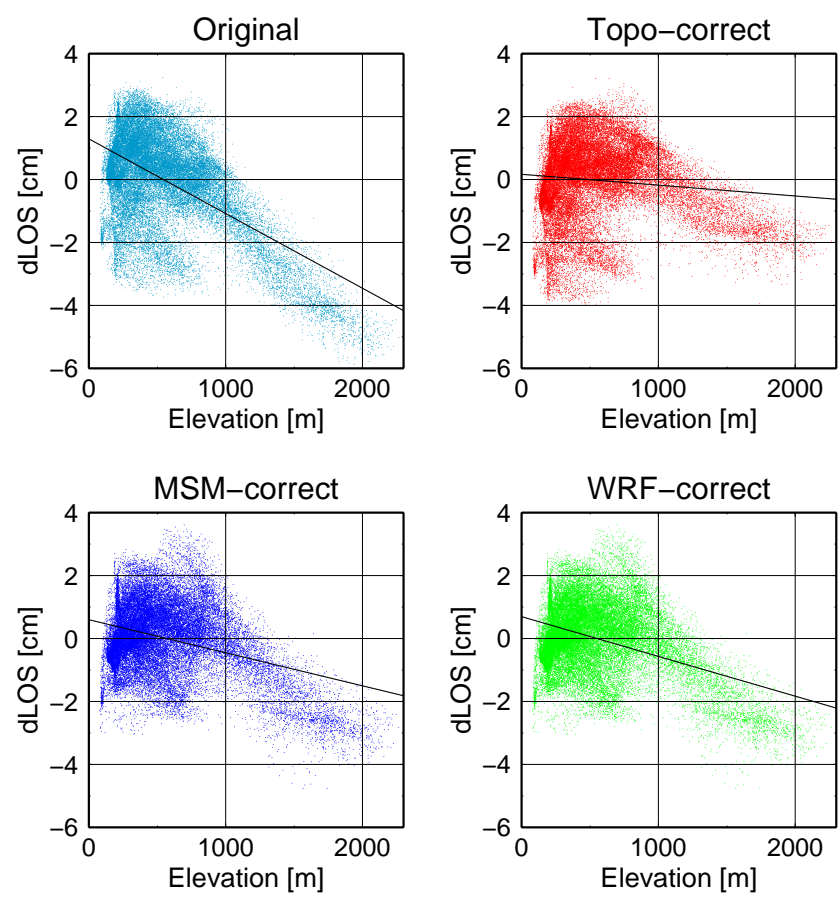

Fig. 6 Variation of the phase with elevation of the surface and the approximated line (black line) in D2 (see Table S1). (Upper left) Scatter plot of the original interferogram. (Upper right) That of the DEM-based corrected interferogram. (Bottom left) That of the MSM-based corrected interferogram. (Bottom right) That of the WRF-based corrected interferogram.

based NWM outputs make the original non-corrected data even "noisier"; the same results are also found in the WRF-based corrections (23 interferograms in Figs. 8 and 9$)$.

We consider that the initial and boundary conditions for the numerical integration are not accurate and precise enough to fully take advantage of the potential performance of the WRF model. Even if the WRF's spatial resolution is set to be $1-\mathrm{km}$ and we compute the tropospheric state at any desired instant, the initial and boundary conditions are still based on the MSM-based low resolution data. As pointed out by Jolivet et al. (2011), while "stratified" tropospheric delay could be significantly reduced with the NWM output, it is not easy to correct for the "non-stratified" (heterogeneous) tropospheric delay because of its poor spatio-temporal resolution. Our study also supports this point to some extent (Figures 8 and 9); the phase dependence with elevation is reduced in 21 pairs corrected with MSM and 29 pairs with WRF. However, the phase dependence with elevation increases in 29 pairs corrected with MSM and 23 pairs with WRF. It could be because the climate of the case area (Hokkaido, Japan) is humid and variable throughout the year, making the task more challenging than in less variably humid parts of the world. In order to generate better results with the WRF-based approach, we will need much higherresolution tropospheric data both spatially and temporally, which would surely be possible in the future.

\section{Summary}

We applied three types of tropospheric delay corrections to 54 interferograms formed from 26 SAR images. If the expected deformation signals are not closely correlated with the topography, and if the "stratified" tropospheric phases are dominant, we found that the simple DEM-based approach will be more effective to correct for the tropospheric phases than using corrections based on the weather models, because the DEM-based approach fit a better model than any other forward models. If one aims to detect small amplitude deformation signals that are correlated with the topography, the NWM-based approach is preferable, and the DEMbased approach should be carefully applied. However, our results indicate that the NWM-based approach is not able to substantially reduce the tropospheric signals when the topography-uncorrelated signals are dominat-
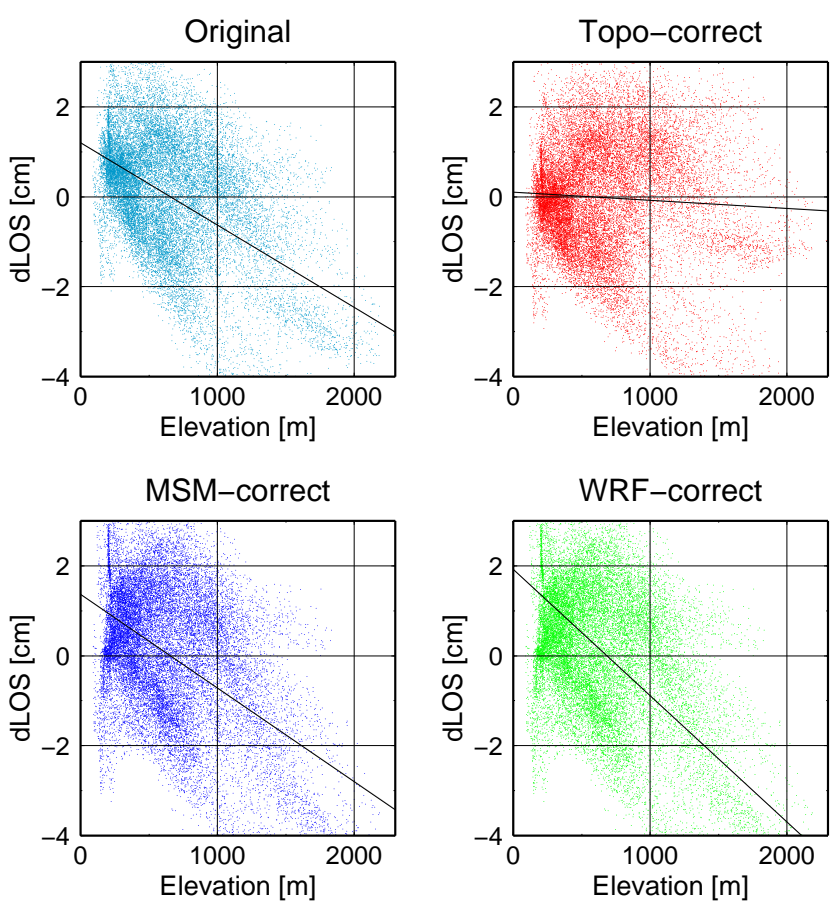

Fig. 7 Variation of the phase with elevation of the surface and the approximated line (black line) in A12 (see Table S1). (Upper left) Scatter plot of the original interferogram. (Upper right) That of the DEM-based corrected interferogram. (Bottom left) That of the MSM-based corrected interferogram. (Bottom right) That of the WRF-based corrected interferogram. 


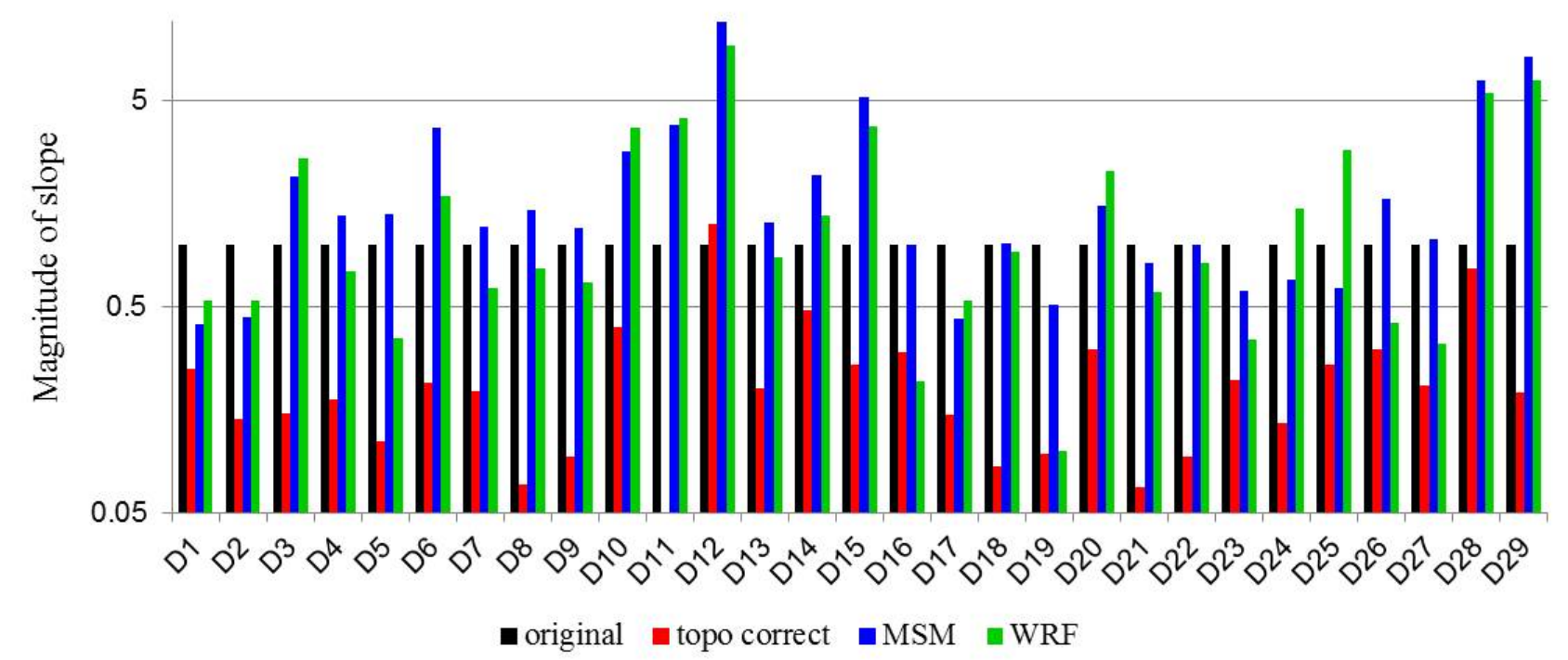

Fig. 8 The slope magnitude for all 29 descending interferograms with and without the tropospheric delay corrections. Slopes of each interferogram are normalized by that of the original interferogram. The red bar in D11 is absent due to the very small value (0.02).

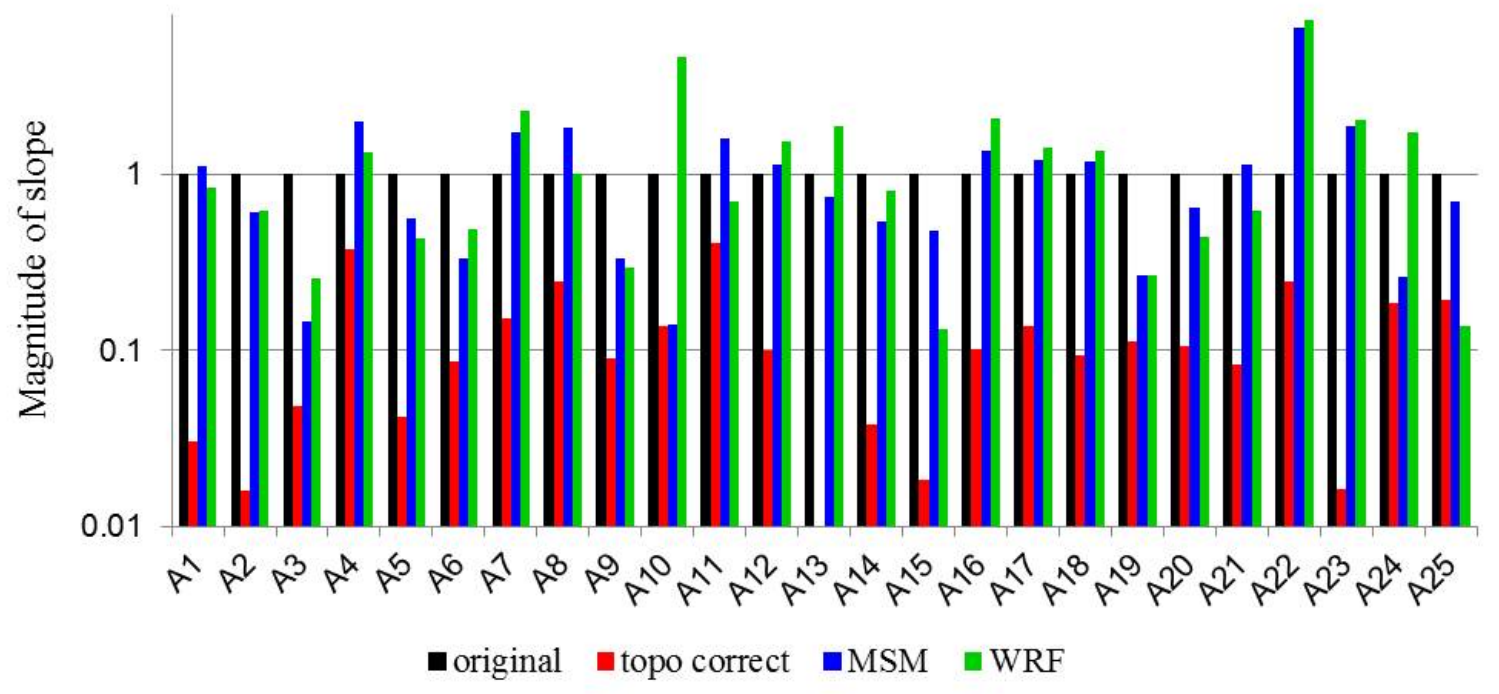

Fig. 9 The slope magnitude for all 25 ascending interferograms with and without the tropospheric delay corrections. Slopes of each interferogram are normalized by that of the original interferogram. The red bar in A13 is absent due to the very small value (0.00467).

ing. We consider that further improvements are necessary in the initial and boundary values for the high spatial-temporal resolution NWM so that the NWM outputs can be helpful in the tropospheric delay correction.

\section{Acknowledgement}

This study was supported by the Ministry of Education, Culture, Sports, Science and Technology of Japan, un- der its Observation and Research Program for Prediction of Earthquakes and Volcanic Eruptions. PALSAR level 1.0 data in this study were provided from the PALSAR Interferometry Consortium to Study our Evolving Land surface (PIXEL) under cooperative research contracts between the Earthquake Research Institute, the University of Tokyo and JAXA. The ownership of PALSAR data belongs to JAXA and the Ministry of Economy, Trade and Industry. The MSM data were acquired from GPV Archive Site (http://database.rish.kyoto-u.a c.jp/arch/jmadata/) managed by the Research Insti- 
tute for Sustainable Humanosphere, Kyoto University. We acknowledge three anonymous reviewers for their helpful comments.

\section{References}

1. Bevis, M., S. Businger, S. Chiswell, T. A. Herring, R. A. Anthes, C. Rocken, and R. H. Ware, GPS meteorology: Mapping zenith wet delays onto precipitable water, J. Appl. Meteor., 33, 379-386 (1994)

2. Berardino, P., G. Fornaro, R. Lanari, and E. Sansostic, A New Algorithm for Surface Deformation Monitoring Based on Small Baseline Differential SAR Interferograms, IEEE. Trans. Geosci. Remote Sensing, 40 (11), 2375-2383 (2002)

3. Beauducel, F., P. Briole, and J. Froger, Volcanowide fringes in ERS synthetic aperture radar interferograms of Etna (1992-1998): Deformation or tropospheric effect?, J. Geophys. Res., 105(B7), 16391-16402, doi:10.1029/2000JB900095 (2000).

4. Bürgmann, R., D. Schmidt, R. M. Nadeau, M. d'Alessio, E. Fielding, D. Manaker, T. V. McEvilly, and M. H. Murray, Earthquake potential along the northern Hayward fault, California, Science, 289, 1178-1182, doi:10.1126/science.289.5482.1178 (2000).

5. Cavalié, O., C. Lasserre, M. P. Doin, G. Peltzer, S. Jianbao, X. Xiwei, and Z. K. Shen, Measurement of interseismic strain across the Haiyuan fault (Gansu, China), by InSAR, Earth Planet. Sci. Lett., 275, 246-257 (2008)

6. Chan, S. T., T. F. Chan, and W. K. Wong, An Intercomparison of WRF-ARW and JMA-NHM Performance in Prediction of Tropical Cyclones over the South China Sea in 2008, 29th Conference on Hurricanes and Tropical Meteorology, American Meteorological Society, P2.62 (2010).

7. Doin, M., C. Lasserre, G. Peltzer, O. Cavalié, and C. Doubre, Corrections of stratified tropospheric delays in SAR interferometry: Validation with global atmospheric models, J. Appl. Geophys., 69, pp. 35-50 (2009)

8. Elgered, G., Tropospheric radio path delay from groundbased microwave radiometry, in Atmospheric Remote Sensing by Microwave Radiometry, edited by M. A. Janssen, pp. 215-258 (1993)

9. Elliot, J. R., J. Biggs, B. Parsons, and T. J. Wright, InSAR slip rate determination on the Altyn Tagh Fault, northern Tibet, in the presence of topographically correlated atmospheric delays, Geophys. Res. Lett., 35, L12309, doi:10.1029/2008GL033659 (2008).

10. Ferreti, A., C. Prati, and F. Rocca, Nonlinear Subsidence Rate Estimation Using Permanent Scatterers in Differential SAR Interferometry, IEEE. Trans. Geosci. Remote Sensing, 38 (5), 2202-2212 (2000).

11. Foster, J., B. Brooks, T. Cherubini, C. Shacat, S. Businger, and C. Werner, Mitigating atmospheric noise for InSAR using a high resolution weather model., Geophys. Res. Lett., 33, L16304 (2006).

12. Fournier, T., M. E. Pritchard, and N. Finnegan, Accounting for atmospheric delays in InSAR data in a search for long-wavelength deformation in South America, IEEE. Trans. Geosci. Remote Sensing, 99, 1-12, doi:10.1109/TGRS.2011.2139217 (2000).

13. Fujiwara, S., P. A. Rosen, M. Tobita, and M. Murakami, Crustal deformation measurements using repeat-pass JERS 1 synthetic aperture radar interferometry near the Izu Peninsula, Japan, J. Geophys. Res., 103, 2411-2426 (1998).
14. Fujiwara, S., M. Tobita, M. Murakami, H. Nakagawa, and P. A. Rosen, Baseline Determination and Correction of Atmospheric Delay Induced by Topography of SAR Interferometry for Precise Surface Change Detection (in Japanese with abstract and figure captions in English), J. Geod. Soc. Jpn., 45 (4), 315-325 (1999).

15. Furuya, M., K. Mueller, and J. Wahr, Active salt tectonics in the Nedles Distinct, Canyonlands (Utah) as detected by interferometric synthetic aperture radar and point target analysis, J. Geophys. Res., 112, B06418, doi:10.1029/2006JB004302 (2007).

16. Furuya, M., SAR Interferometry, in Encyclopedia of Solid Earth Geophysics, Part 16, 1041-1049, ed. H. Gupta (2011).

17. Goldstein, R. M., H. Engelhardt, B. Kamb, and R. M. Frolich, Sattelite radar interferometry for monitoring icesheet motion - Application to an antarctic ice stream, Science, 262, 1525-1530, doi:10.1126/science.262.5139.1525 (1993).

18. Goldstein, R. M., and C. L. Werner, Radar interferogram filtering for geophysical applications, Geophys. Res. Lett., 25, doi:10.1029/1998GL900033 (1998).

19. Gray, A. L., K. E. Matter, and G. Sofko, Influence of ionospheric electron density fluctuations on satellite radar interferometry, Goephys. Res. Lett., 27, 1451-1454 (2000)

20. Hanssen, R. F., Radar Interferometry: Data interpretation and error analysis, Kluwer Academic Press (2001).

21. Hayashi, S., K. Aranami, and K. Saito, Statistical Verification of Short Term NWP by NHM and WRF-ARW with $20 \mathrm{~km}$ Horizontal Resolution around Japan and Southeast Asia, SOLA, 4, 133-136 (2008).

22. Hobiger, T., R. Ichikawa, Y. Koyama, and T. Kondo, Fast and accurate ray-tracing algorithms for real-time space geodetic applications using numerical weather models, $J$. Geophys. Res., 113, D20302, doi:10.1029/2008JD010503 (2008).

23. Hobiger, T., Y. Kinoshita, S. Shimizu, R. Ichikawa, M. Furuya, T. Kondo, and Y. Koyama, On the importance of accurately ray-traced troposphere corrections for Interferometric SAR data, J. Geodesy, doi:10.1007/s00190-0100393-3 (2010).

24. Hooper, A., H. Zebker, P. Segall, and B. Kampes, A new method for measuring deformation on volcanoes and other natural terrains using InSAR persistent scatterers, Geophys. Res. Lett., 31, L23611, doi:10.1029/2004GL021737 (2004).

25. Jolivet, R., R. Grandin, C. Lasserre, M. -P. Doin, and G. Peltzer, Systematic InSAR tropospheric phase delay corrections from global meteorological reanalysis data, Geophys. Res. Lett., in press (2011).

26. Li, Z., J.-P. Muller, P. Cross, and E. J. Fielding, Interferometric synthetic aperture radar (InSAR) atmospheric correction: GPS, Moderate Resolution Imaging Spectroradiometer (MODIS), and InSAR integration, J. Geophys. Res., 110, B03410, doi:10.1029/2004JB003446 (2005).

27. Li, Z., E. J. Fielding, P. Cross, and J.-P. Muller, Interferometric synthetic aperture radar atmospheric correction: Medium Resolution Imaging Spectrometer and Advanced Synthetic Aperture Radar integration, Geophys. Res. Lett., 33, L06816, doi:10.1029/2005GL025299 (2006).

28. Lin, Y.N., M., Simons, E.A., Hetland, P. Muse, and C. DiCaprio, A Multiscale approach to estimating topographically correlated propagation delays in radar interferograms, Geochem. Geophys. Geosyst., 11, Q09002, doi:10.1029/2010GC003228 (2010).

29. Massonnet, D., M. Rossi, C. Carmona, F. Adragna, G. Peltzer, K. Feigl, and T. Raboute, The displacement field 
of the Landers earthquake mapped by radar interferometry, Nature, 364, 138-142 (1993).

30. Massonnet, D., and K. L. Feigl, Radar interferometry and its application to changes in the earth's surface, Rev. Geophys., 36(4), 441-500, doi:10.1029/97RG03139 (1998).

31. Meyer, F., R. Bamler, N. Jakowski, and T. Fritz, Methods for small scale ionospheric TEC mapping from broadband L-band SAR data, in Proc. IGARSS, Denver, CO, Jul. 31Aug. 4, 3735-3738 (2006).

32. Onn, F., and H. A. Zebker, Correction for interferometric synthetic aperture radar atmospheric phase artifacts using time series of zenith wet delay observations from a GPS network, J. Geophys. Res., 111, B09102, doi:10.1029/2005/JB004012 (2006).

33. Otsuka, A., S. Kobayashi, and H. Seko, A wind-induced delay pattern in SAR interferometry and numerical simulation, J. Jpn. Soc. Photogrammetry and Remote Sensing, 41 (4), 85-98 (2002).

34. Ozawa, T. and S. Shimizu, Atmospheric noise reduction in InSAR analysis using numerical weather model (in Japanese with abstract and figure captions in English), $J$. Geod. Soc. Jpn., 56(4), 137-147 (2010).

35. Puysségur, B., R. Michel, and J. Avouac, Tropospheric phase delay in interferometric synthetic aperture radar estimated from meteorological model and multispectral imagery, J. Geophys. Res., 112, B05419, doi:10.1029/2006JB004352 (2007).

36. Shimada, M., Correction of the Satellite's State Vector and the Atmospheric Excess Path Delay in the SAR Interferometry -An Application to Surface Deformation Detection- (in Japanese with abstract and figure captions in English), J. Geod. Soc. Jpn., 45(4), 327-346 (1999).

37. Shimada, M., Y. Muraki, and Y. Otsuka, Discovery of anomalous stripes over the Amazon by the PALSAR onboard ALOS satellite, in Proc. IEEE IGARSS, Jul. 2008, pp.II-387-II-390 (2008).

38. Simons, S. and P. A. Rosen, Interferometric Synthetic Aperture Radar, in Geodesy, Treatise on Geophysics, vol. 3, 391-446, ed. T. Herring, Elsevier (2009).

39. Skamaroch, W. C., J. B. Klemp, J. Dudhia, D. O. Gill, D. M. Barker, M. G. Duda, X. Y. Huang, W. Wang, and J. G. Powers (2008): A Description of the Advanced research WRF Version 3, NCAR technical note, NCAR/TN-475+STR. (Available at http://www.mmm.ucar.edu/wrf/users/docs/arw_v3.pdf)

40. Thayer, G. D., An improved equation for the radio refractive index of air, Radio Sci., vol. 9, no. 10, pp. 803-807 (1974)

41. Wadge, G., P. W. Webley, I. N. James, R. Bingley, A. Dodson, S. Waugh, T. Veneboer, G. Puglisi, M. Mattia, D. Baker, S. C. Edwards, S. J. Edwards, and P. J. Clarke (2002): Atmospheric models, GPS and InSAR measurements of the tropospheric water vapour field over Mount Etna, Geophys. Res. Lett., 29, 2002GL015159.

42. Wadge, G., M. Zhu, R. J. Holley, I. N. James, P. A. Clark, C. Wang, and M. J. Woodage, Correction of atmospheric delay effects in radar interferometry using a nested mesoscale atmospheric model, J. Appl. Geophys., 72, pp. 141-149 (2010)

43. Zebker, H. A., P. A. Rosen, and S. Hensley (1997): Atmospheric artifacts in interferometric synthetic aperture radar surface deformation and topographic maps, J. Geophys. Res., 102, 7547-7564. 